Review began 02/11/2022 Review ended 02/28/2022 Published 03/01/2022

๑) Copyright 2022

$\mathrm{Uz}$ Zaman et al. This is an open access article distributed under the terms of the Creative Commons Attribution License CCBY 4.0., which permits unrestricted use, distribution, and reproduction in any medium, provided the original author and source are credited.

\section{Temporal Bone Cholesteatoma: Typical Findings and Evaluation of Diagnostic Utility on High Resolution Computed Tomography}

Sameeh Uz Zaman ${ }^{1}$, Varsha Rangankar ${ }^{1}$, Krishnarjun Muralinath ${ }^{1}$, Viraj Shah ${ }^{1}$, Gowtham $\mathrm{K}^{2}$, Rishikesh Pawar $^{2}$

1. Radiology, Dr. D. Y. Patil Medical College, Hospital \& Research Centre, Pune, IND 2. Otolaryngology - Head and Neck Surgery, Dr. D. Y. Patil Medical College, Hospital \& Research Centre, Pune, IND

Corresponding author: Krishnarjun Muralinath, krishnarjun918@gmail.com

\section{Abstract}

\section{Background}

Pre-operative assessment of middle ear cholesteatoma is a must for assessing the disease's location, extent, and complication, and high-resolution computed tomography (HRCT) is the modality of choice. Therefore, this study aims to assess the common signs of cholesteatoma on HRCT and its diagnostic ability.

\section{Methods}

Fifty patients with suspected cholesteatoma were considered for the study, which was carried out on an Ingenuity Core 128 slice CT scanner (Philips, Amsterdam, Netherlands). The bilateral temporal bones of 50 patients were assessed for soft tissue density and associated findings. The number of temporal bones with soft tissue density was then correlated with intra-operative and histopathological examinations (HPE).

\section{Results}

Out of 100 temporal bones, 63 were diseased, and 37 were normal temporal bones. Epitympanum/Prussak's space was the most involved site with soft tissue density seen in 60/63 (95.2\%) diseased temporal bones, followed by aditus ad antrum and mesotympanum, which was seen in 51/63 (80.9\%) diseased temporal bones. The majority of the soft tissue lesions were non-dependent, accounting for $43 / 63$ (68.2\%) of the diseased temporal bones. Bony erosions were seen in 54/63 (85.7\%) and bony expansion in 35/63 (55.5\%) of the diseased temporal bones. HRCT showed a sensitivity of $100 \%$, specificity of $88.1 \%$, a positive predictive value (PPV) of $92.1 \%$, a negative predictive value (NPV) of $100 \%$, and accuracy of $95 \%$ for detection of cholesteatoma.

\section{Conclusion}

HRCT of the temporal bone precisely demonstrates cholesteatoma's location, extent, and bony changes. Therefore, it has exceptional sensitivity, high specificity, and accuracy in diagnosing cholesteatoma.

Categories: Otolaryngology, Radiology, Infectious Disease

Keywords: chronic suppurative otitis media, external auditory canal cholesteatoma, hrct temporal bone, high resolution computed tomography, cholesteatoma

\section{Introduction}

Chronic otitis media (COM) is a stage of ear disease in which there is a long-term infection of the middle ear cleft, which includes the Eustachian tube, the middle ear, and the mastoid. In this stage, there is the presence of a ruptured tympanic membrane and drainage [1]. Clinically, there are two main types of chronic suppurative otitis media: one with no cholesteatoma, known as the "safe" type, and one with cholesteatoma, known as the "unsafe" type [2].

Cholesteatoma is, in simple terms, "skin in the wrong place." Cholesteatoma consists of an outer lining composed of stratified squamous epithelium, an inner keratin debris content within the cholesteatoma sac which is in turn secreted by the epithelium, and an external peri-matrix that secretes bone destroying proteolytic enzymes [3].

The clinical diagnosis of cholesteatoma is made by otoscopic examination, and the gold standard is histopathology or during operative exploration. On the other hand, imaging measures for diagnosis, such as high-resolution computed tomography (HRCT) and magnetic resonance imaging (MRI), are utilized. HRCT is the imaging modality of choice for diagnosing cholesteatoma due to its ability to assess the gross extent and erosion of the fine bony architecture of the temporal bone. Hence, HRCT is an indispensable pre-operative 
necessity [3].

On HRCT, cholesteatoma appears as a soft-tissue "mass-like" density in the middle ear cavity and mastoid antrum with associated signs of mass effect in the form of surrounding smooth bony erosions and expansion cavities [4]. The likelihood of cholesteatoma increases when the soft tissue opacity is non-dependent [5]. The absence of soft tissue density in the middle ear mastoid complex rules out cholesteatoma. However, HRCT cannot characterize the soft tissue density and differentiate cholesteatoma from inflammatory/granulation tissue or scar tissue [6]. The purpose of the present study is to enumerate the typical signs of cholesteatoma on HRCT and evaluate the diagnostic ability of HRCT for temporal bone cholesteatoma.

\section{Materials And Methods \\ Case selection}

The present study was a descriptive observational research between September 2019 and August 2021. It was conducted after permission was granted from the Institutional Ethics Sub-Committee (IESC) at Dr. D.Y. Patil Medical College, Hospital and Research Center, Pune, MH, India, with approval number IESC/PGS/2019/173. Every patient took written informed consent. Fifty patients were referred from the Otorhinolaryngology and Head and Neck Surgery department with suspicion of primary cholesteatoma or recurrent cholesteatoma in post-operative cases based on clinical presentation, otoscopic examination and audiometric tests. Patients of all ages were included in our study. The patients with soft tissue density in the temporal bone suspected to be cholesteatoma on HRCT underwent surgery. Surgery was performed by two otorhinolaryngologists with experience of 15 and 16 years respectively. The final diagnosis was arrived at utilizing surgical and histopathological validation.

\section{Imaging technique}

HRCT imaging was obtained on Philips Ingenuity Core (Philips, Amsterdam, Netherlands), a 128 slice CT scanner. The high-resolution CT images were obtained with $20 \times 0.625$ collimation and $0.8 \mathrm{~mm}$ thickness using $320 \mathrm{mAs}$, and $120 \mathrm{kVp}$ with ultrathin image reconstruction using the high-resolution bone algorithm in the axial plane with $0.5 \mathrm{~mm}$ section thickness, $0.01 \mathrm{~mm}$ increments, and a FOV of 100 , with a matrix size of $512 \times 512$. This isotropic image data was used to obtain coronal and sagittal reformatted images. The image interpretation was made using a 3D workstation.

\section{Imaging evaluation}

In our 50-patient sample, the bilateral temporal bones of each patient were assessed. Total 100 highresolution temporal bone studies of the 50 patients were evaluated in detail in axial and reformatted coronal and sagittal planes. The HRCT findings in both the temporal bones were noted under the headings: aeration status of mastoid, sclerosis of mastoid air cells, location of the soft tissue lesion, the extent of soft tissue lesion, bony expansion (e.g., widened aditus ad antrum, mastoid cavity), bony erosions (e.g., scutum, middle ear walls, mastoid air cells), ossicular chain status (malleus, incus, stapes), tegmen tympani erosion, sinus plate erosion, facial canal dehiscence, and other findings. All the HRCT studies were assessed by a single radiologist with an experience of 16 years with special focus on neuroradiology and head and neck imaging.

\section{Statistical analysis}

Sensitivity, specificity, positive predictive value (PPV), negative predictive value (NPV), and accuracy of HRCT in diagnosing cholesteatoma were calculated with a 95\% confidence interval by correlating them to the gold standard-HPE/Intraoperative results. The statistical analysis was done using Microsoft Excel 2007 and Statistical Package for Social Sciences (SPSS) version 26 (IBM Corp., Armonk, NY, USA).

\section{Results}

The 50 patients with chronic suppurative otitis media (CSOM) or probable post-operative cholesteatoma recurrence included in this research had an average age of $25 \pm 10.8$, ranging from eight to 65 years. The majority of patients (20\%) were aged between 30 and 40 years. There was an equal number of males and females (25 patients each), with no gender preference. Our research sample's most common clinical symptom was purulent ear discharge, found in 42 cases (84\%). Hearing loss (64\%) and otalgia were common complaints (46\%). Most of the patients (60\%) had history of recurrent upper respiratory tract infection. Only one patient had a history of ear trauma.

On HRCT, 35 patients (70\%) had soft tissue density in the unilateral temporal bone, and 14 patients (28\%) had soft tissue density in bilateral temporal bones.

In our study, the bilateral temporal bones of each patient, i.e., 100 temporal bones in 50 patients, were assessed on HRCT. The temporal bones that showed soft tissue density was labeled as diseased temporal bone on HRCT. Out of 100 temporal bones, 63 were diseased, and 37 were normal temporal bones. Of the 63 diseased temporal bones, 54 were primary diseased, and nine had recurrent disease post-operatively. One clinically symptomatic patient had no soft tissue on HRCT. 


\section{Cureus}

Epitympanum/Prussak's space was the most involved site with soft tissue density seen in 60/63 (95.2\%) diseased temporal bones, followed by aditus ad antrum and mesotympanum, which was seen in 51/63 (80.9\%) diseased temporal bones. Soft tissue density was present in the mastoid antrum and air cells in 46/63 (73\%) diseased temporal bones and hypotympanum in 20/63 (31.7\%) diseased temporal bones. External auditory canal (EAC), sinus tympani, facial canal recess, and eustachian tube were the least involved in 10/63 (15.8\%), 5/63 (7.9\%), 4/63 (6.3\%), and 1/63 (1.5\%) diseased temporal bones, respectively (Table 1).

\begin{tabular}{|c|c|c|}
\hline Involvement of individual parts & Number & Percentage \\
\hline Epitympanum/Prussak space & 60 & 95.2 \\
\hline Mesotympanum & 51 & 80.9 \\
\hline Hypotympanum & 20 & 31.7 \\
\hline Aditus ad Antrum & 51 & 80.9 \\
\hline Mastoid Antrum and air cells & 46 & 73 \\
\hline Sinus Tympani & 5 & 7.9 \\
\hline Facial canal recess & 4 & 6.3 \\
\hline Eustachian Tube & 1 & 1.5 \\
\hline EAC & 10 & 15.8 \\
\hline
\end{tabular}

\section{TABLE 1: Soft tissue density at different locations in diseased temporal bones on HRCT}

EAC: external auditory canal, HRCT: high-resolution computed tomography

The most common typical site (Table 2) of soft tissue density involvement was attico-antral in 32/63 (50.7\%) diseased temporal bones, followed by extensive holotympanic seen in 19/63 (30.1\%) and attic in 10/63 (15.8\%) diseased temporal bones (Figure 1A, 1B). Conversely, the least common locations were isolated mesotympanum and EAC (Figure 1C), each seen in 1/63 (1.5\%) diseased temporal bone.

\begin{tabular}{|c|c|c|}
\hline Involvement & Number & Percentage \\
\hline Attico-antral & 32 & 50.7 \\
\hline Holotympanic & 19 & 30.1 \\
\hline Attic & 10 & 15.8 \\
\hline Mesotympanum only & 1 & 1.5 \\
\hline EAC & 1 & 1.5 \\
\hline
\end{tabular}

TABLE 2: Typical locations of soft tissue density in the diseased temporal bones on HRCT.

EAC: external auditory canal, HRCT: high-resolution computed tomography 


\section{Cureus}
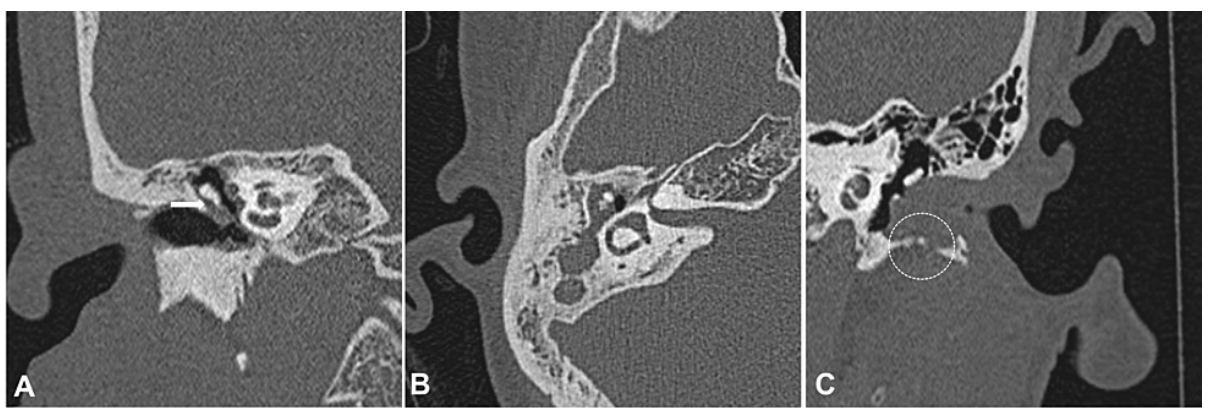

FIGURE 1: HRCT images demonstrating locations of cholesteatoma. Coronal reformatted HRCT image of the right temporal bone (A) shows non-dependent soft tissue in Prussak's space (white arrow) proved as cholesteatoma. Axial HRCT image of right temporal bone showing attico-antral cholesteatoma (B). Coronal reformatted HRCT image demonstrating soft tissue density lesion and bony erosions in the left EAC (C), a rare cholesteatoma location.

EAC: external auditory canal, HRCT: high-resolution computed tomography

The most consistent imaging finding of CSOM was the loss of aeration and sclerosis of mastoid air cells and was present in 61/63 (96.8\%) diseased temporal bones. The majority of the soft tissue lesions were nondependent (Figure $2 A$ ), accounting for $43 / 63$ (68.2\%) of the soft tissue density lesions in the diseased temporal bones. The other common findings suggesting CSOM with cholesteatoma were bony erosions seen in 54/63 (85.7\%) diseased temporal bones followed by bony expansion (Figure $3 A$ ) in 35/63 (55.5\%) of the diseased temporal bones. Lateral semicircular fistula (Figure $3 E$ ) and petrous apicitis were present in 3/63 (4.7\%) and 1/63 (1.5\%) of the diseased temporal bones (Table 3). Incus was the most common structure eroded, seen in $38 / 63$ (63\%) of the diseased temporal bones. The other common structures eroded were malleus (Figure $2 B$ ) seen in 37/63 (58.7\%), scutum (Figure 3B) seen in 35/63 (55.5\%), stapes (Figure 3D) in $30 / 63$ (47.6\%), tegmen tympani (Figure $2 A$ ) in 24/63 (38\%), and facial canal (Figure 3B) in 22/63 (34.9\%) out of the diseased temporal bones. The least affected structures were lateral semicircular canal and bony EAC, seen in 3/63 (4.7\%) diseased temporal bones each (Table 4).
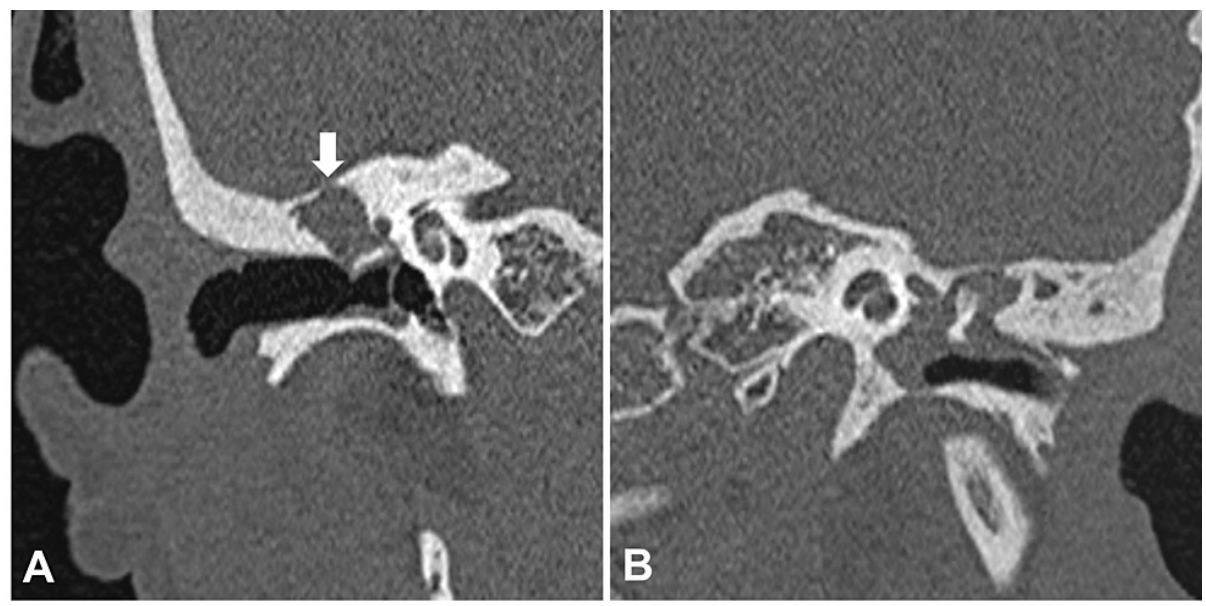

FIGURE 2: A reformatted coronal CT image of a patient which shows a non-dependent soft tissue density in the right middle ear $(A)$ which is causing erosion of the tegmen tympani (white arrow). A coronal reformatted CT image of a patient which shows a soft tissue density in the left middle ear (B) which is causing erosion and deossification of the head of malleus. 


\section{Cureus}
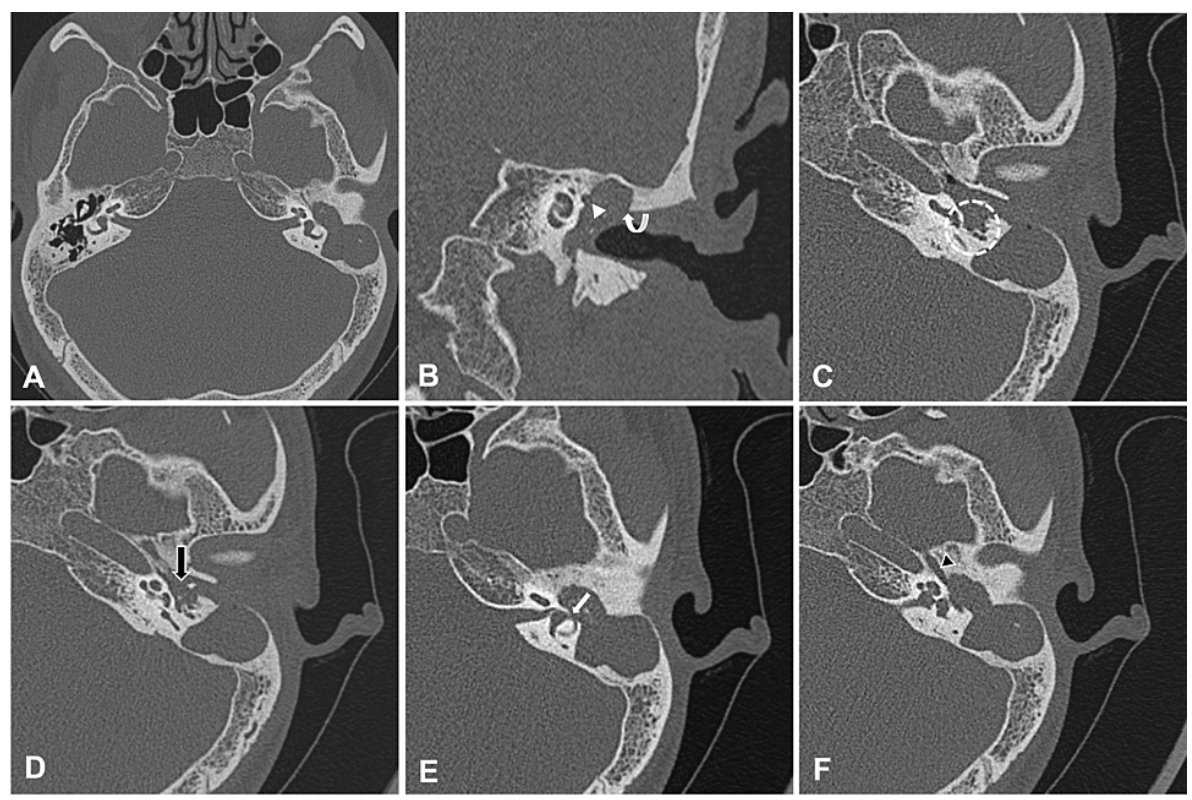

FIGURE 3: Axial HRCT image (A) showing a large well-defined soft tissue density in the left middle ear extending into the aditus and mastoid antrum, causing bony expansion and destruction of the malleus and incus with loss of ice cream cone appearance. Coronal reformatted CT image in the same patient (B) shows soft tissue density occupying the epitympanum, mesotympanum, and hypotympanum. It is seen causing erosion of the scutum (curved arrow) and inferior wall of the horizontal portion of the facial canal (arrowhead). Axial HRCT image (C) showing soft tissue (encircled) is seen extending into the sinus tympani and facial canal recesses. Axial HRCT image showing the destruction of stapes (D) and mastoid cortex dehiscence with thinning of dural sinus plate (D). Axial HRCT image (E) shows a focal defect in the wall of the lateral semicircular canal (white arrow) suggestive of perilymphatic fistula. Soft tissue extension into the eustachian tube (black arrowhead) was also seen (F).

HRCT: high-resolution computed tomography

\begin{tabular}{|c|c|c|}
\hline HRCT signs & Number & Percentage \\
\hline Loss of aeration and sclerosis of mastoid air cells & 61 & 96.8 \\
\hline Bony erosions & 54 & 85.7 \\
\hline Non-dependent soft tissue density lesions & 43 & 68.2 \\
\hline Bony expansion & 35 & 55.5 \\
\hline Lateral semicircular canal fistula & 3 & 4.7 \\
\hline Petrous Apicitis & 1 & 1.5 \\
\hline
\end{tabular}

TABLE 3: HRCT signs of CSOM and cholesteatoma in diseased temporal bones. 


\section{Cureus}

\begin{tabular}{|c|c|c|}
\hline Location of bony erosions & Number & Percentage \\
\hline Scutum & 35 & 55.5 \\
\hline Malleus & 37 & 58.7 \\
\hline Incus & 38 & 60.3 \\
\hline Stapes & 30 & 47.6 \\
\hline Tegmen tympani thinning/erosion & 24 & 38 \\
\hline Facial canal & 22 & 34.9 \\
\hline Mastoid cortex dehiscence & 4 & 6.3 \\
\hline Lateral semi-circular canal dehiscence & 3 & 4.7 \\
\hline Bony EAC & 3 & 4.7 \\
\hline
\end{tabular}

TABLE 4: Location of bony erosions in the diseased temporal bones.

EAC: external auditory canal

Out of 63 temporal bones with soft tissue density, 58 temporal bones were proven as cholesteatoma, and five were false positive as confirmed on intraoperative/HPE findings. These false-positive cases were granulation tissue (two), cholesterol granuloma (one), non-cholesteatomatous otitis media (one), and wax (one) (Figure 4).
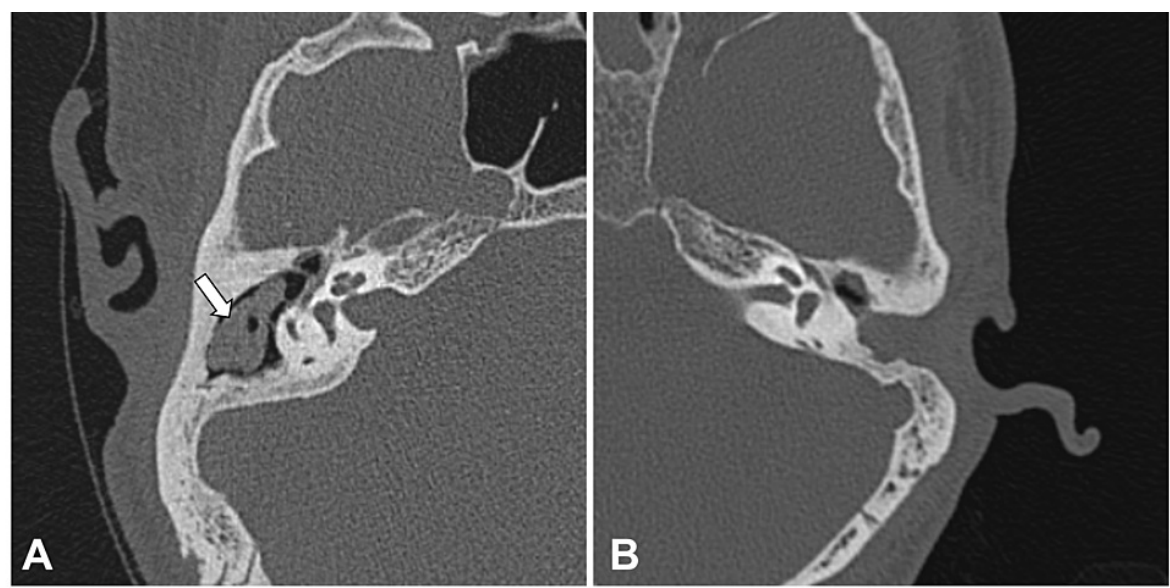

FIGURE 4: Axial right temporal HRCT image shows soft tissue density in the right post-operative mastoid cavity (A) suspected to be cholesteatoma was proven as wax (white arrow). Axial HRCT image of another patient showing soft tissue density in the left middle ear and post-operative mastoid cavity (B), which was proven to be granulation tissue.

HRCT: high-resolution computed tomography

In our study, HRCT temporal bone had a sensitivity of $100 \%$ (with CI of $93.8 \%-100 \%$ ), specificity of $88.1 \%$ (with CI of $74.4 \%-96.0 \%$ ), PPV of $92.1 \%$ (with CI of $82.4 \%-97.4 \%$ ), NPV of $100 \%$ (with CI of $90.5 \%-100 \%$ ) and accuracy of $95 \%$ (with CI of $88.7 \%-98.4 \%$ ) for diagnosis cholesteatoma with intra-operative/HPE correlation.

\section{Discussion}

The ability of HRCT to define CSOM and its complication, mainly the cholesteatoma prior to otologic 
surgery, is well established [7]. HRCT is especially useful in detecting early erosive changes in the ossicles and hidden soft tissue, particularly in the smaller regions like sinus tympani and facial canal recess. HRCT can not characterize and differentiate the cholesteatomatous soft tissue from other pathologies like granulation tissue, scar tissue, wax, granuloma, etc. [6,8]. However, HRCT is superior to magnetic resonance imaging (MRI), positron emission tomography (PET) CT and Technetium-99m studies as it is readily available, economical and gives the detailed bony architecture of the middle ear in the backdrop of CSOM [3]. Though MRI can help characterize the soft tissue, it lacks the spatial resolution and ability to assess bony changes in the temporal bone.

Out of the 100 temporal bones of the 50 patients studied, 63 temporal bones showed soft tissue density and were labeled as diseased temporal bones on HRCT. Of 63 diseased temporal bones, 54 (85.7\%) temporal bones were primarily diseased, and nine (14.3\%) temporal bones suspected post-operative recurrence.

In our study, the unilateral disease was seen in 35 (70\%) patients, and bilateral disease was seen in 14 (28\%) patients, which is in line with the study done by Gomaa et al., which revealed bilateral disease in 16 (28.5\%) out of 50 patients [9].

Most of these soft tissue density lesions in our study were non-dependent, i.e., $68.2 \%$ of the diseased temporal bones, a vital sign of cholesteatoma. Rai, in their study of 50 patients, reported 45 (90\%) patients having non-dependent soft tissue [10]. The different percentage of non-dependent soft tissue in our study was likely due to more extensive soft tissue progression and holotympanic involvement seen in 19 diseased temporal bones.

In the present study, the most common location of soft tissue was the epitympanum/Prussak space seen in 60 (95.2\%) of the 63 diseased temporal bones. Other common locations were aditus ad antrum and mesotympanum seen in 51 (80.9\%) and mastoid antrum seen in 46 (73\%) of the diseased temporal bones. Furthermore, Jacob et al. performed an HRCT study on 30 cases of CSOM with cholesteatoma, which showed soft tissue density in the epitympanum in 22 (73.3\%) of patients followed by mesotympanum 17 (56.6\%) and aditus ad antrum in $16(53.3 \%)$ of the patients [11].

In the study done by Gomaa et al., according to the typical locations of soft tissue density found in CSOM, the majority of the cases had soft tissue density as extensive holotympanic in 18 (32.1\%) patients, followed by attic in 16 (28.5\%) patients, attico-antral in 12 (21.4\%) and mesotympanum in 10 (17.8\%) patients [9]. However, in our study, the most common typical site was attico-antral in 32 (50.7\%) diseased temporal bones, followed by extensive holotympanic seen in 19 (30.1\%) diseased temporal bones. Attic involvement was seen in 10 (15.8\%) diseased temporal bones, and one (1.5\%) had mesotympanum and external auditory canal involvement. The EAC cholesteatoma, a rare location for cholesteatoma, was seen in one case and showed surrounding bony erosion, differentiating it from its main differential of keratosis obturans [3].

The loss of aeration and sclerosis of mastoid air cells seen in 61 (96.8\%) diseased temporal bones was the most consistent finding of CSOM in our study. In an HRCT evaluation of 64 patients of unsafe type of CSOM by Gaurano et al., 100\% of the patients were found to have affected mastoid air cells [12].

Bone erosion was seen in 54 (85.7\%) out of 63 diseased temporal bones. Incus was found to be the most common ossicle eroded in 38 (60.3\%) diseased temporal bones, followed by malleus in 37 (58.7\%) and stapes in 30 (47.6\%) diseased temporal bones. A similar trend was reported by Manik et al. in an HRCT study of 50 symptomatic patients, which showed Incus to be the most commonly eroded in 35 (70\%) patients, followed by malleus in 21 (42\%) and stapes in 17 (34\%) patients [13]. Scutum was eroded in 35 (55.5\%) diseased temporal bones in the present study. Rai, in their research, reported scutum erosion in 23 (65\%) patients [10]. The present research saw tegmen tympani erosion in 24 (38\%) diseased temporal bones. In an HRCT study done by Jamal et al. in 50 symptomatic patients, tegmen tympani erosion was seen in 13 (30\%) patients [14]. There was no case of dural sinus plate erosion encountered in our study as it is a rare complication of CSOM/cholesteatoma [9-13].

In the present study, bony expansion was seen in 35 (55.5\%) diseased temporal bones. However, in the study done by Gaurano et al., bony expansion was seen in 59 (92\%) cases [12]. We found the facial canal dehiscence in 22 (34.9\%) diseased temporal bones. The study by Jamal et al. reported facial canal dehiscence in 15 (30\%) out of 50 patients [14]. In our study, mastoid cortex dehiscence was seen in four (6.3\%) diseased temporal bones, similar to Rai, who reported mastoid cortex dehiscence in four (8\%) patients [10]. Our study noted lateral semicircular canal fistula in three (4.7\%) diseased temporal bones. This observation was seen to concur with an HRCT study done by Dashottar et al. in 50 symptomatic patients, which reported lateral semicircular canal fistula in two (4\%) patients [15].

HRCT could detect soft tissue lesions of any size with no false-negative cases in the present study. Hence sensitivity turned out to be $100 \%$. However, five patients were falsely diagnosed with cholesteatoma, as HRCT could not surely differentiate cholesteatoma from granulation tissue (two cases), cholesterol granuloma (one case) and non-cholesteatomatous otitis media (one case). These are some of the common causes for false-positive cases on HRCT as reported in the literature $[3,4,6]$. One case with soft tissue in the 
post-operative mastoid cavity suspected as cholesteatoma was found to be impacted wax on surgery. The open mastoid cavity in canal wall down procedures are known to have excessive wax build-up due to altered epithelial migration [16]. We found the specificity of HRCT in diagnosing cholesteatoma to be $88.1 \%$ in correlation with the intra-operative and HPE findings. Mitra et al. reported a sensitivity and specificity of $100 \%$ for the diagnosis of cholesteatoma in their HRCT study of 100 patients [17]. Payal et al. performed an HRCT temporal bone study on 60 patients and got a sensitivity of $89.6 \%$ and specificity of $100 \%$ [18]. Reddy et al. found a sensitivity of $92 \%$ and a specificity of $66 \%$ in their HRCT study of 25 patients of CSOM [19].

\section{Limitations}

The sample size of our study was only 50 patients. This could be why complications of CSOM like intracerebral complications and dural sinus thrombosis were not seen. Furthermore, the signs of cholesteatoma seen on HRCT, such as mastoid air cells sclerosis, bony erosions, lateral semicircular canal (LSCC) fistula, bony expansion, facial canal, and mastoid cortex dehiscence, were not correlated with intraoperative findings in our study. Thus individual sensitivity and specificity of these findings could not be measured. In addition, 14 patients (28\%) had bilateral CSOM disease in our study. Hence, findings in the affected temporal bones were considered instead of affected patients, and due to this, our study could not be classically correlated with other studies which evaluated affected patients. The present study was primarily focussed on the HRCT imaging evaluation of cholesteatoma, and did not assess the underlying pathological mechanism.

\section{Conclusions}

HRCT temporal bone is an invaluable diagnostic tool for detecting early cholesteatoma, tiny and cholesteatoma in hidden locations. It helps assess the bony integrity, detects various associated complications with high accuracy, and provides a roadmap for the surgeon during cholesteatoma surgery. In addition, HRCT also helps assess the recurrent disease in the post-operative temporal bones. Thus, HRCT provides a diagnostic capability with exceptional sensitivity and considerably high specificity in detecting cholesteatoma and the state of the bone structures in the temporal bone.

\section{Additional Information \\ Disclosures}

Human subjects: Consent was obtained or waived by all participants in this study. Institutional Ethics SubCommittee, Dr. D.Y. Patil Medical College, Hospital and Research Center, Pune - 411018 issued approval IESC/PGS/2019/173. Animal subjects: All authors have confirmed that this study did not involve animal subjects or tissue. Conflicts of interest: In compliance with the ICMJE uniform disclosure form, all authors declare the following: Payment/services info: All authors have declared that no financial support was received from any organization for the submitted work. Financial relationships: All authors have declared that they have no financial relationships at present or within the previous three years with any organizations that might have an interest in the submitted work. Other relationships: All authors have declared that there are no other relationships or activities that could appear to have influenced the submitted work.

\section{References}

1. Head K, Chong LY, Bhutta MF, et al.: Topical antiseptics for chronic suppurative otitis media . Cochrane Database Syst Rev. 2020, 1:CD013055. 10.1002/14651858.CD013055.pub2

2. Seiden AM, Tami TA, Penssak ML, Cotton RT, Gluckman JL: Otorhinolaryngology, The Essentials. Seiden AM (ed): Thieme, New York; 2002.

3. Baráth K, Huber AM, Stämpfli P, Varga Z, Kollias S: Neuroradiology of cholesteatomas. AJNR Am J Neuroradiol. 2011, 32:221-9. 10.3174/ajnr.A2052

4. Mafee MF, Levin BC, Applebaum EL, Campos CF: Cholesteatoma of the middle ear and mastoid . Otolaryngol Clin North Am. 1988, 21:265-8. 10.1016/S0030-6665(20)31539-5

5. Liu DP, Bergeron RT: Contemporary radiologic imaging in the evaluation of middle ear-attic-antral complex cholesteatomas. Otolaryngol Clin North Am. 1989, 22:897-909. 10.1016/s0030-6665(20)31366-9

6. Jackler RK, Dillon WP, Schindler RA: Computed tomography in suppurative ear disease: a correlation of surgical and radiographic findings. Laryngoscope. 1984, 94:746-52. 10.1288/00005537-198406000-00004

7. Chee NC, Tan TY: The value of preoperative high resolution CT scans in cholesteatoma surgery . Singapore Med J. 2001, 2:155-9.

8. Mafee MF, Kumar A, Yannias DA, Valvassori GE, Applebaum EL: Computed tomography of the middle ear in the evaluation of cholesteatomas and other soft-tissue masses: comparison with pluridirectional tomography. Radiology. 1983, 148:465-72. 10.1148/radiology.148.2.6867344

9. Gomaa MA, Abdel Karim AR, Abdel Ghany HS, Elhiny AA, Sadek AA: Evaluation of temporal bone cholesteatoma and the correlation between high resolution computed tomography and surgical finding. Clin Med Insights Ear Nose Throat. 2013, 6:21-8. 10.4137/CMENT.S10681

10. Rai T: Radiological study of the temporal bone in chronic otitis media: prospective study of 50 cases . Indian J Otol. 2014, 20:48. 10.4103/0971-7749.131865

11. Jacob A, Sreedhar S, Choolakkaparambu A, Anwar S, Bashir, Nalakath K: Utility of high resolution computed tomography in pre-operative evaluation of cholesteatoma. Int J Otorhinolaryngol Head Neck Surg. 2020, 6:1278. 10.18203/issn.2454-5929.ijohns20202781 


\section{Cureus}

12. Gaurano JL, Joharjy IA: Middle ear cholesteatoma: characteristic CT findings in 64 patients . Ann Saudi Med. 2004, 24:442-7. 10.5144/0256-4947.2004.442

13. Manik S, Dabholkar Y, Bhalekar S, Velankar H, Chordia N, Saberwal A: Sensitivity and specificity of highresolution computed tomography (HRCT) of temporal bone in diagnosing cholesteatoma and its correlation with intraoperative findings. Indian J Otolaryngol Head Neck Surg. 2021, 73:25-9. 10.1007/s12070-02001892-z

14. Jamal S, Rahman A, Mohan C, Srivastava A: Is pre-operative HRCT temporal bone findings consistent with tympano-mastoid surgical findings. Int J Health Clin Res. 2020, 3:104-9.

15. Dashottar S, Bucha A, Sinha S, Nema D: Preoperative temporal bone HRCT and intra-operative findings in middle ear cholesteatoma: a comparative study. Int J Otorhinolaryngol Head Neck Surg. 2018, 5:77. 10.18203/issn.2454-5929.ijohns20185070

16. Khemani S, Singh A, Lingam RK, Kalan A: Imaging of postoperative middle ear cholesteatoma . Clin Radiol. 2011, 66:760-7. 10.1016/j.crad.2010.12.019

17. Mitra M, Thakare P, Monteiro B, Amle D: The role of high-resolution computerized tomography as a diagnostic tool in the evaluation and planning of management of patients with chronic otitis media. Muller J Med Sci Res. 2021, 12:6. 10.4103/mjmsr.mjmsr_30_21

18. Payal G, Pranjal K, Gul M, Mittal MK, Rai AK: Computed tomography in chronic suppurative otitis media: value in surgical planning. Indian J Otolaryngol Head Neck Surg. 2012, 64:225-9. 10.1007/s12070-011-0325-

19. Sirigiri RR, Dwaraknath K: Correlative study of HRCT in attico-antral disease . Indian J Otolaryngol Head Neck Surg. 2011, 63:155-8. 10.1007/s12070-011-0162-5 